\title{
Confronting jet quenching with jet grooming: jet mass distributions in heavy ion collisions
}

\author{
Yang-Ting Chien*广 \\ Center for Theoretical Physics, Massachusetts Institute of Technology, Cambridge, MA 02139, \\ U.S.A. \\ E-mail: ytchien@mit.edu
}

\begin{abstract}
In this proceeding we present a calculation of jet mass distributions for small-radius jets in proton-proton and heavy ion collisions using soft-collinear effective theory (SCET). A processindependent groomed jet mass function is defined which captures the soft-collinear radiation inside jets. The factorization expression simplifies significantly, allowing one to calculate jet mass distributions for jets in any hard process with the corresponding jet cross section. Additional contributions from initial and final state radiation as well as underlying events are discussed. With the medium-induced splitting functions calculated using SCET with Glauber gluon interactions, the medium correction to jet mass distributions is incorporated consistently within the resummation framework. We provide calculations of jet mass distributions for inclusive jets and jets recoiling against a prompt photon or a $\mathrm{Z}$ boson, and we compare them with PYTHIA simulations of pp collisions and with currently available measurements in pp and AA collisions at the LHC.
\end{abstract}

International Conference on Hard and Electromagnetic Probes of High-Energy Nuclear Collisions 30 September - 5 October 2018

Aix-Les-Bains, Savoie, France

\footnotetext{
* Speaker.

${ }^{\dagger}$ The author would like to thank the Hard Probes 2018 organizers for the hospitality and support.
} 

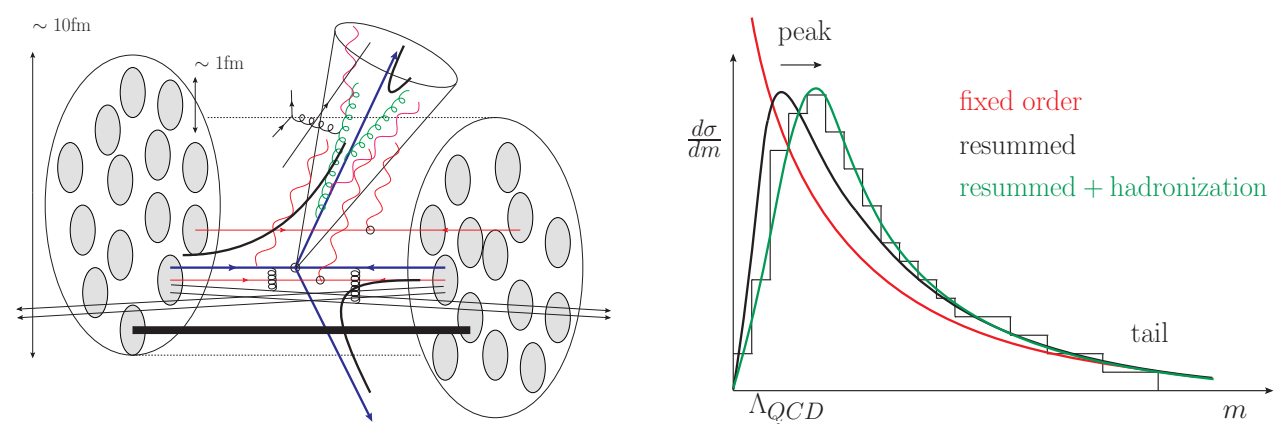

Figure 1: Left panel: Various radiation contributions inside a jet in a heavy ion collision. Right panel: The necessity of all-order resummation and the effect of hadronization to the jet mass distribution.

\section{Introduction}

A precise understanding of the redistribution of jet energy is among the priorities of jet physics in heavy ion collisions. Various proposed jet quenching mechanisms capture the picture of energy loss by transporting the jet energy to wider angle. Possible contributions from medium response to jets also affect the energy distribution. Therefore designing jet substructure observables which can probe aspects of jet-medium interaction is vital for revealing possible, novel signatures of jet quenching. On the other hand, techniques which help clean up jets - generally referred to as jet grooming - have been introduced to mitigate background soft radiation in order to enhance the signal sensitivity. Groomed jet observables with different grooming parameters thus have different sensitivities to radiation inside jets, giving the opportunities to test the details of jet quenching mechanisms and rigorously determine the medium properties.

In this proceeding we exploit the connection between jet quenching and jet grooming and present a calculation for groomed jet mass distributions. The jet mass is a classic jet observable which is sensitive to both energetic, collinear radiation as well as soft, wide-angle radiation (represented by the green and red lines, respectively, in the left panel of Fig. 1). There are many possible sources of soft radiation, including final-state and initial-state radiation, underlying events activity correlated or uncorrelated with the hard collision, medium-induced radiation and medium response. The calculation of jet mass distribution requires all-order resummation of logarithmically-enhanced terms, as well as modeling non-perturbative contributions (right panel of Fig. 1). We use softcollinear effective theory (SCET) to factorize the cross section, and to resum, via the renormalization group evolution, the logarithmic corrections appearing among different scales (left panel of Fig. 2). We focus on the medium modification contribution caused by induced radiation via Glauber interaction [1]. We use the soft drop [2] grooming procedure to remove soft, wide-angle radiation (right panel of Fig. 2). Below we summarize the formalism for calculating the soft drop jet mass distribution and present some preliminary results.

\section{Framework}

Following the framework developed in $[3,4,5,6]$ for various jet substructure calculations, at next-to-leading logarithmic (NLL) accuracy one can define a process-independent groomed jet 

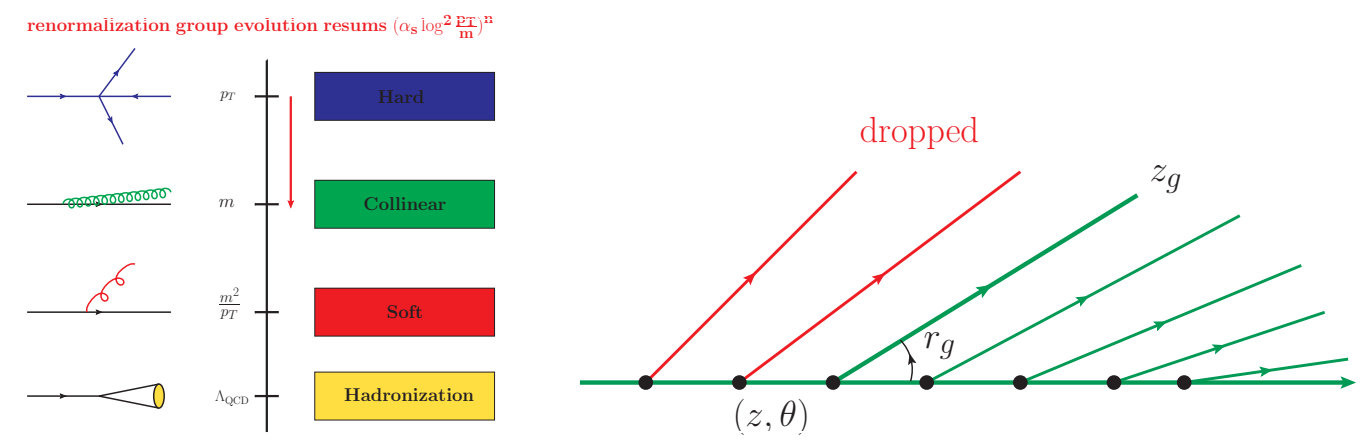

Figure 2: Left panel: Factorization of jet mass in SCET and resummation performed using renormalization group evolution. Right panel: The angular-ordered tree in the soft drop procedure.

mass function $J^{S D}\left(m^{2}, \mu\right)$ which captures all the soft-collinear radiation inside jets $(i=q, g$ labeling quark-initiated or gluon-initiated jets),

$$
J_{i}^{S D}\left(m^{2}, \mu\right)=\int d p^{2} d k J_{i}\left(p^{2}, \mu\right) S_{i}^{S D}\left(k, R, z_{c u t}, \mu\right) \delta\left(m^{2}-p^{2}-2 E_{J} k\right),
$$

where $S_{i}^{S D}\left(k, R, z_{\text {cut }}, \mu\right)=S_{i}^{C}\left(k, R, z_{\text {cut }}, \mu\right) S_{i}^{G}\left(R, z_{\text {cut }}, \mu\right)$ can be further factorized. At fixed-order, the jet mass function can be directly calculated using the collinear splitting function,

$$
J_{i}^{S D}\left(m^{2}, \mu\right)=\sum_{j, k} \int_{P S} d x d k_{\perp} \mathscr{P}_{i \rightarrow j k}\left(x, k_{\perp}\right) \delta\left(m^{2}-M^{2}\left(x, k_{\perp}\right)\right) \Theta_{\text {alg. }} \Theta_{S D} .
$$

Here $M^{2}\left(x, k_{\perp}\right)=\frac{k_{\perp}^{2}}{x(1-x)}$ is the jet mass measurement function, while $\Theta_{\mathrm{k}_{\mathrm{T}}}=\Theta\left(E_{J} R x(1-x)-\right.$ $\left.k_{\perp}\right)$ and $\Theta_{S D}=\Theta\left(E_{J} R x(1-x)\left(\frac{x}{z_{c u t}}\right)^{1 / \beta}-k_{\perp}\right)$ are the jet algorithm and soft drop phase space constraints, respectively. The medium modification can also be included using medium-induced splitting functions. The full jet mass distribution can then be calculated by weighing the groomed jet mass functions with the corresponding jet cross sections,

$$
\frac{d \sigma}{d m^{2}}=\sum_{i=q, g} \int_{P S} d p_{T} d y \frac{d \sigma^{i}}{d p_{T} d y} P_{i}^{S D}\left(m^{2}, \mu\right), \text { where } P_{i}^{S D}\left(m^{2}, \mu\right)=\frac{J_{i}^{S D}\left(m^{2}, \mu\right)}{J_{u n}^{i}(\mu)} .
$$

Note that the ratio $P_{i}^{S D}\left(m^{2}, \mu\right)$ between the jet mass function and the unmeasured jet function is renormalization-group invariant and can be interpreted as the probability distribution of the jet mass for jets initiated by parton $i$.

\section{Preliminary results}

Having outlined the framework of our calculation, we present some preliminary results to compare with PYTHIA simulations and to currently available measurements. The left panel of Fig. 3 shows the comparison between NLL SCET calculations and ALICE measurements [7] of inclusive charged jet mass distributions in $2.76 \mathrm{TeV} \mathrm{PbPb}$ and $5.02 \mathrm{TeV} \mathrm{pPb}$ collisions. In the calculation we relate the total jet mass to the charged jet mass assuming isospin symmetry. The calculation does not yet include non-perturbative effects therefore the jet mass is systematically lower than 

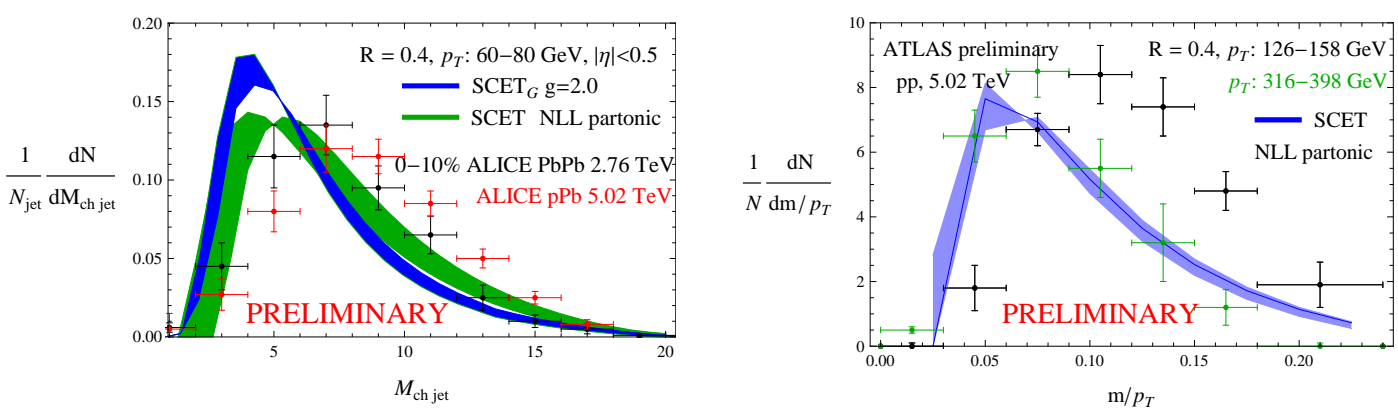

Figure 3: Left panel: NLL SCET calculations (assuming isospin symmetry) and ALICE measurements [7] of inclusive charged jet mass distributions in $2.76 \mathrm{TeV} \mathrm{PbPb}$ and $5.02 \mathrm{TeV}$ pPb collisions. Right panel: NLL SCET calculation and ATLAS measurement [8] of inclusive jet mass over transverse momentum ratio in $5.02 \mathrm{TeV}$ pp collisions.

the measured values. However, we can see that the medium modifies the jet mass towards smaller values. The right panel of Fig. 3 shows the comparison between SCET calculation and ATLAS measurement [8] of inclusive jet mass over jet transverse momentum $\left(\mathrm{m} / \mathrm{p}_{T}\right)$ distribution in 5.02 $\mathrm{TeV}$ pp collisions. We see that the partonic calculation agrees well with the measured distribution for jets at higher $p_{T}$ where non-perturbative effects are small.
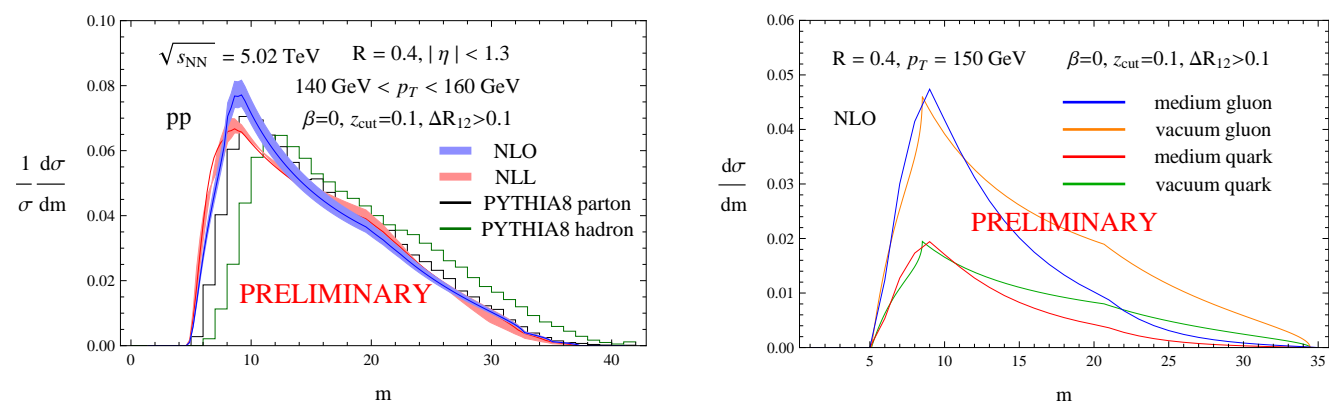

Figure 4: Left panel: PYTHIA simulations compared to the NLO and NLL+NLO SCET calculations of inclusive soft drop jet mass distribution in $5.02 \mathrm{TeV}$ pp collisions. Right panel: NLO vacuum and medium contributions to inclusive soft drop jet mass distribution in SCET.

The left panel of Fig. 4 shows the comparison of NLO and NLL+NLO SCET calculation with PYTHIA simulations at parton and hadron level for inclusive soft drop jet mass distribution in 5.02 $\mathrm{TeV}$ pp collisions. We see that the parton level results agree quite well, and that hadronization affects the distribution significantly in all regions. The right panel shows the NLO vacuum and medium contributions and suggests that the small jet mass region is enhanced by the medium.

The CMS measurement imposes a $\Delta R_{12}$ cut to constrain the angle between two soft drop branches therefore removing the Sudakov peak region [9]. The formalism allows us to calculate the jet mass distribution for jets from arbitrary hard scattering process. Fig. 5 shows the NLL SCET calculation (left panel) of jet mass distribution for $Z+$ jet events in $7 \mathrm{TeV}$ pp collisions and compare with CMS measurements [10] (right panel). We see the very good agreement which further confirms the validity of the framework. 

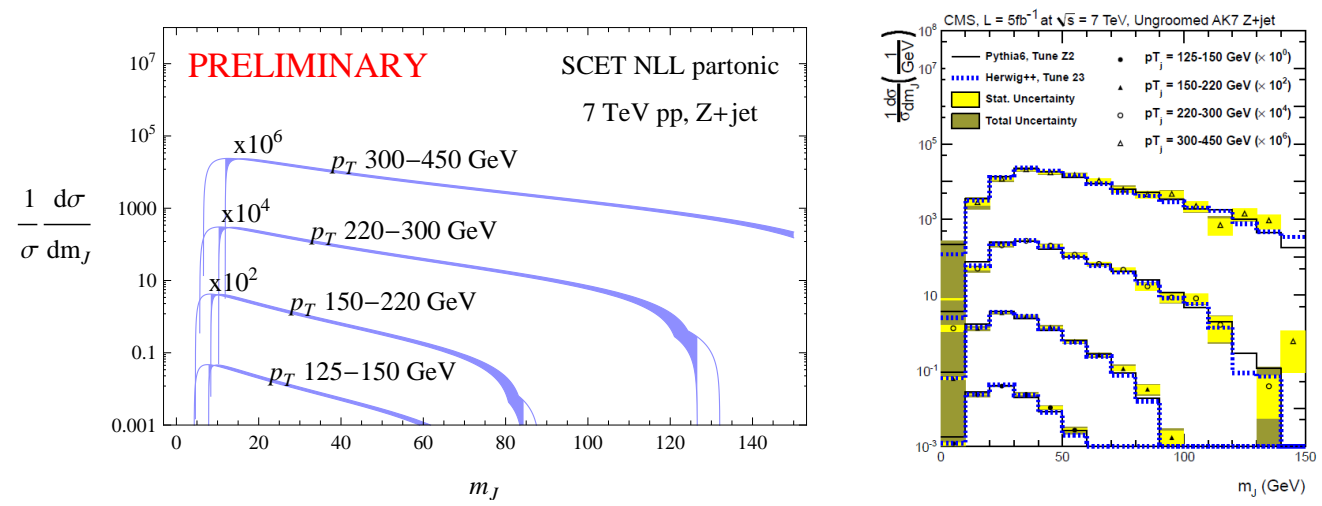

Figure 5: NLL SCET calculation (left panel) and CMS measurements [10] (right panel) of jet mass distribution in $Z+$ jet events in $7 \mathrm{TeV}$ pp collisions.

\section{Conclusions}

Jet mass contains rich information of the radiation inside jets, and jet grooming gives a concrete strategy to probe specific regions which can reveal possible jet quenching features. We provide an effective field theory framework to systematically resum logarithmically-enhanced contributions and calculate the jet mass distribution at NLL accuracy for any arbitrary hard scattering process with jets in the final state. This work provides a baseline for future precision jet substructure studies.

\section{References}

[1] G. Ovanesyan and I. Vitev, An effective theory for jet propagation in dense QCD matter: jet broadening and medium-induced bremsstrahlung, JHEP 1106, 080 (2011)

[2] A. J. Larkoski, S. Marzani, G. Soyez and J. Thaler, Soft Drop, JHEP 1405, 146 (2014)

[3] H. n. Li, Z. Li and C.-P. Yuan, QCD resummation for light-particle jets, Phys. Rev. D 87, 074025 (2013)

[4] Y. T. Chien, R. Kelley, M. D. Schwartz and H. X. Zhu, Resummation of Jet Mass at Hadron Colliders, Phys. Rev. D 87, no. 1, 014010 (2013)

[5] Y. T. Chien and I. Vitev, Jet Shape Resummation Using Soft-Collinear Effective Theory, JHEP 1412, 061 (2014)

[6] Y. T. Chien and I. Vitev, Towards the understanding of jet shapes and cross sections in heavy ion collisions using soft-collinear effective theory, JHEP 1605, 023 (2016)

[7] S. Acharya et al. [ALICE Collaboration], First measurement of jet mass in $\mathrm{PbPb}$ and $\mathrm{pPb}$ collisions at the LHC, Phys. Lett. B 776, 249 (2018)

[8] The ATLAS collaboration [ATLAS Collaboration], Measurement of $R=0.4$ jet mass in $\mathrm{Pb}+\mathrm{Pb}$ and pp collisions at $\sqrt{s_{\mathrm{NN}}}=5.02 \mathrm{TeV}$ with the ATLAS detector, ATLAS-CONF-2018-014.

[9] A. M. Sirunyan et al. [CMS Collaboration], Measurement of the groomed jet mass in PbPb and $p p$ collisions at $\sqrt{s_{\mathrm{NN}}}=5.02 \mathrm{TeV}$, JHEP 1810, 161 (2018)

[10] S. Chatrchyan et al. [CMS Collaboration], Studies of jet mass in dijet and W/Z + jet events, JHEP 1305, 090 (2013) 